\title{
VIEW: A CONCEPT FOR AN IMMERSIVE VIRTUAL REALITY IMAGE INTERPRETATION WORKBENCH
}

\author{
Alexander Streicher, Julien Hoffmann and Wolfgang Roller \\ Fraunhofer IOSB, Karlsruhe, Germany
}

\begin{abstract}
Virtual Reality (VR) and serious gaming can increase the learning motivation and outcome in image interpretation. This paper presents the concept of an VR game-like assistance system called Virtual Reality Image Exploitation Workspace (VIEW). It can be used to evaluate the learning-supporting immersion or presence level of image interpreters while solving typical interpretation tasks. The problem statement is centered on the realization aspects of a VR assistance system for image interpretation which provides a high level of immersion. This work combines the setting of a real serious game for formal image interpretation training with virtual reality to increase the immersion level of the users. Enhanced image quality and organizational requirements from professional image interpretation impose additional complexity on VR for image interpretation. The presented VIEW concept deals with multiple aspects in respect to e-learning, professional training and virtual reality for image interpretation. Preliminary evaluation results from a realized prototype show the feasibility and user acceptance of the VIEW concept.
\end{abstract}

\section{KEYWORDS}

Virtual Reality, Immersion, Image Interpretation, Serious Games, Assistance Systems

\section{INTRODUCTION}

Serious Gaming and Virtual Reality (VR) have long since been an active topic in professional training (Freina and Ott, 2015; Mazuryk and Gervautz, 1996). With the currently available commercial off-the-shelf hardware these training applications and the research therein have seen a staggering increase (Ganbatte DevBlog, 2018). VR is especially well suited for immersive experiences. In combination with gaming, virtual worlds provide users with rich playable environments, welcoming playing, testing and learning.

The application area of our work is aerial and satellite image interpretation. The interpretation of aerial and satellite images poses a wide range of challenges because of different imagery sensor types, difficult image material or unknown objects. Professional image interpretation is done in various fields, ranging from the microscopic level in medicine, e.g., diagnostics in histopathology, to the macroscopic level of aerial or satellite images, e.g., civilian applications like search-and-rescue, agriculture, oceanography, or military intelligence acquisition (Roller et al., 2013). Image interpretation requires the detection, recognition, identification and analysis of structures and objects, according to a given task based on optical, infrared and radar imagery. Besides highly developed assets, sensors and image exploitation systems, the success of a reconnaissance mission depends on the interpreter's analysis performance. Special face-to-face training courses blended with technology-enhanced learning tools, such as e-Learning courses, simulations and serious games, ensure that image analysts are qualified to a high standard. Our application scenarios focus on intelligent assistance and e-learning systems that help users to perform their work effectively and efficiently.

An immersive virtual environment can promote the motivation for continuous usage and therefore it can ultimately promote learning. A VR system with a high immersion capacity facilitates the sensation of presence, i.e., the sensation of "being there" (Riva et al., 2003). The notion of immersion and presence often go hand in hand and several terms have been coined with subtle differences, e.g., immersion, presence, involvement, emotional response or degree of interest. In this paper, we use immersion as the technical form factor that features presence. Besides the motivational aspects of immersion or presence, there are further technical benefits for image interpretation, such as the efficient display of 3D data or spatial objects, ease of spatial cognition (Hubona and Shirah, 2005), virtually infinite space, or dislocated collaboration. 
In the past huge efforts were needed to make spatial data tangible, e.g., by producing statuary, or by using 3D glasses for anaglyphic stereoscopic printing (red-green) or for 3D monitors. With VR one can immerse in a virtual environment and directly experience 3D.

In aerial image interpretation, the cognitive experience of the spatial data can have a considerable impact on the quality of the interpretation reports, e.g., when spatial relations could be experienced in more detail and with a natural access to reference scales. For example, VR can easily mediate topographic map data.

To achieve immersion we follow gamification and digital game based learning paths (Prensky, 2001). The combination of gamification and VR is obvious - as the entertainment industry proves with VR games numbered in the millions (Ganbatte DevBlog, 2018). In a professional (or serious) context, VR can be combined with aspects of serious gaming. A serious game is a digital game not just for entertainment but with at least one characterizing goal (Dörner et al., 2016), e.g., learning or training. In digital game based learning (Prensky, 2001) the game design is highly oriented at the target group and the learning objectives. A high acceptance for the game's assets and game mechanics promote the acceptance by the users which enables immersion, which in turn promotes the intrinsic motivation for playing or training, which positively influences flow and ultimately could increase the learning efficacy (Connolly et al., 2012).

The superordinate research question (and application scenario) of our work is how to optimally train and support professional image interpreters. This paper specifically addresses how to increase the immersion level in assistance systems for image interpretation using VR. The adjacent subquestion is how to design VR systems for image interpretation that fulfill the quality requirements regarding image fidelity, high resolution and optimal usability in a professional working context.

This paper presents the concept study and prototype realization of the Virtual Reality Image Exploitation Workspace (VIEW), a VR assistance as well as a training system for image interpreters. The realization targets commercial VR hardware and implementation in a VR capable game engine. The concept offers answers to some challenges that are specific for VR for image interpretations, e.g., image fidelity, interaction paradigms or display of high-resolution imagery.

\section{RELATED WORK}

VR itself has long been an active research topic with multiple industrial appliances (Nomura and Sawada, 2001). The dome-like display for urban planning by Nomura et al. influenced our concept (Nomura and Sawada, 2001). Of further inspiration has been the work of (Streitz et al., 2001); they analyzed how to build interactive office environments by integrating various display types. In some parts, this has influenced our concept, e.g., the design and placement of our virtual displays and interaction elements. Other driving work has been from the hierarchy of needs in VR development by Cronin (Cronin, 2015), with special focus on the factors interpretability (sense) and usefulness (value).

For image interpretation VR has been used for visualization geoscience data, e.g, by Lin et al. with a VR geoscience image interpretation workplace (Lin and Loftin, 1998). We followed also their Closed World Interaction (CWI) concept that influences the complexity - or rather simplicity - of intuitive VR interface design with low affordance. Such interaction concepts should be followed to benefit from VR visualization advantages (Loftin and Nelson, 2000), although no VR interaction standards exist yet (Malaika, 2015).

The advantages of using VR for visualizing high resolution image data in an immersive virtual environment has been presented by (Billen et al., 2008; Loftin and Nelson, 2000). This is in line with other work which show that geoscience can benefit from VR interaction possibilities (Furumura and Chen, 2004; Ohno and Kageyama, 2007). A Study by Lackey et al. (Lackey et al., 2016) show how positive VR experiences can reduce stress and workload. Such kind of immersive environments can optimally utilize the brain's cognitive capacity to process visual information (Hubona and Shirah, 2005). 


\section{VIRTUAL IMAGE EXPLOITATION WORKSPACE (VIEW)}

VIEW is a concept for a VR assistance system for image interpreters which incorporates immersive elements suitable $^{1}$ for professional image interpretation. In this context an operational image interpretation assistance system, ViLand (Figure 1\}, has been used as a base model for VIEW. Such systems offer tools for image navigation, manipulation and annotation as well as specific reporting functionalities, e.g., standardized reporting processes and formats.

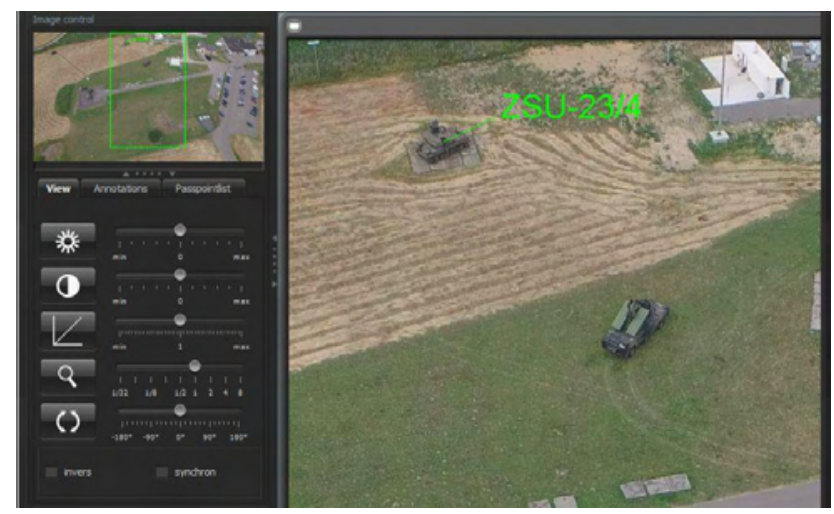

Figure 1. Assistance system ViLand for image interpretation and reporting

\subsection{Virtual Image Display (VID)}

For image interpretation, the Virtual Image Display (VID) is apparently the most important aspect. Based on requirements analysis with experts on aerial and satellite image interpretation the demands for a VR display comprise: high resolution, image fidelity, multi-channel imagery and image manipulation.

The VID is a virtual monitor where the imagery data is displayed, navigated or modified; it is VIEW's primary user interface (UI) component. In the game engine this is technically a dynamic texture. Various manifestations of the texture's underlying model have been discussed and evaluated (Figure 2): flat or curved plane, quarter-, half- or full dome, or 360 degree panorama. The objective is to maximize the visible display for quick and sound recognition. Although this leads to a half- or full dome or a 360 degree panorama, it is not the typical type of content in image interpretation. Also it necessitates ample head movements which hinders quick recognition and has the risk of fatigue (Hoppe, 2016). Dome-like approaches (Figure 2) inherently impose too much unacceptable vertical and horizontal distortions on the image data. Image interpreters require a reliable and undistorted display. Although a flat plane does not impose distortions effects on the texture (no curvature), it proved difficult for the users to view content in the corners for a big VID. This leads to a compromise: a curved VID (Figure 2). The curved model requires less head movements and can cover the peripheral vision.
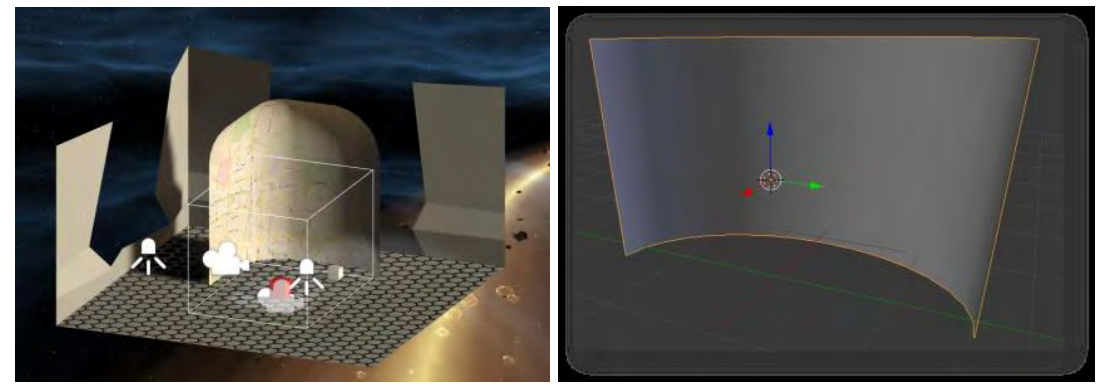

Figure 2. Tested display concepts; (left) dome-like display with avatar in the center; (right) curved display

\footnotetext{
${ }^{1}$ Suitable in this context means that the professional or serious context is being considered, i.e., less entertainment in favor of productivity.
} 


\subsubsection{High Resolution}

The "virtual resolution" should be as high as possible to display as much content as possible. Professional image interpreters often use multi-monitor workstations for maximum display of image data as well as instant access to tools. Especially in aerial or satellite image interpretation with huge images or maps one monitor is used for maps, one for the tools (e.g., reporting tool), and the remaining monitors for displaying and working with one or multiple images, e.g., navigating, annotating, modifying, etc.

The challenge is to display huge image data that does not fit into the GPU memory. A typical aerial image easily exceeds 50 megapixels and $1500 \mathrm{MB}$. Today's game engines and GPU allow a maximum size of $8192 \times 8192$ pixels for a texture. Therefore, the image has either to be scaled (loosing details) or split up.

The solution approach is to use tiled rendering. We propose to use a software architecture (Figure 3 ) with a mapserver, e.g., GeoServer ${ }^{2}$, and a slippy map framework, e.g., UnitySlippyMap ${ }^{3}$ for Unity3D. This enables the integration of various sources, e.g., vector maps, (geo-referenced) images, markers, etc.

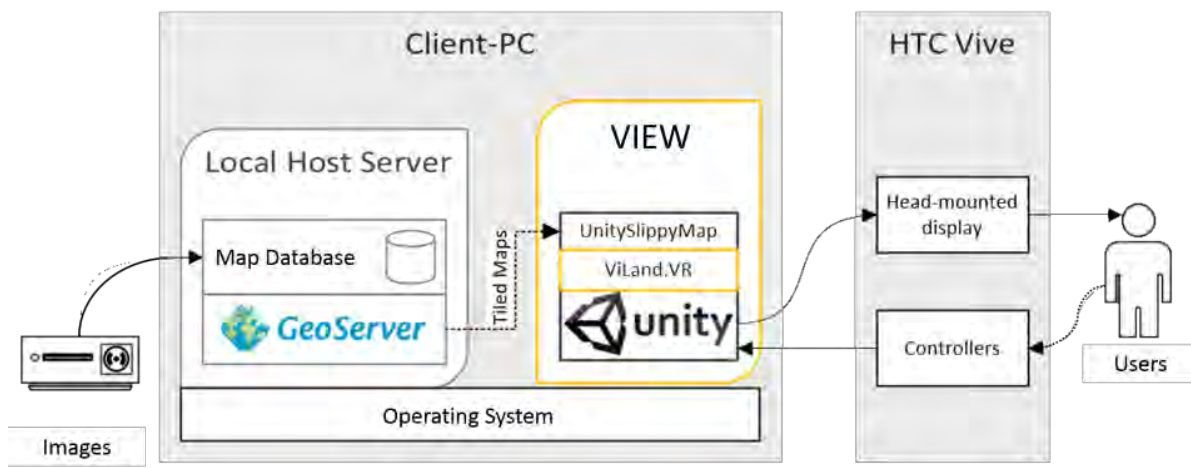

Figure 3. VIEW software architecture using Unity3D game engine and GeoServer for image or map tiling

\subsubsection{Image Fidelity}

The correct and sound image rendering and display is essential; the user acceptance towards the interpretation system highly correlates with the subjectively recognizable display quality. Professional image interpretation implements technical measures to ensure image fidelity, e.g., special hardware with higher color range (i.e., 10 bit per color channel instead of consumer 8 bit) or calibrated displays. Software measures include lossless compression or accountable metadata management, e.g., for validated documentation processes. The geospatial server approach solves this for our VIEW concept. In the rendering engine, image modifications (e.g., compression) must be disabled, and textures must have uniform, neutral-colored lighting.

Critical and not yet solved issues concerns the VR hardware, in particular the head-mounted display (HMD). Whereas continuous development shows an increase in the HMD display resolutions, some negative effects can still be seen (Anthes et al., 2016), like the latency, screen-door effect, lens distortions, ring effects or light rays. We propose to lay the focus on the usability and the subjective acceptance level for the target group, e.g., by a pre-study and requirement analysis.

\subsubsection{Multi Channel Imagery Display}

A further requirement is the possibility to display various types of imagery data, i.e., electro-optical (EO), infrared, radar and hyperspectral. Ideally, video streams should be supported as well. Important is the overlay aspect and geo-referencing. It must be possible to display the very same object or area in different visual channels but with identical alignment. For an area this means that the location of the overlaid images must be identical. The mapserver approach solves this by providing access to different map or image sources which can be combined with overlay layers. In VR/VIEW the VID is just the window to the mapserver and the layer switching is propagated to the server by means of standard protocols (e.g., web map service, WMS).

\footnotetext{
${ }^{2}$ www.geoserver.org, open source mapserver server for geospatial data sharing.

${ }^{3}$ https://github.com/jderrough/UnitySlippyMap
} 


\subsection{Interaction Concepts}

Especially in a professional working context the interaction paradigms must be swift and without risk of fatigue, and, of course, the typical usability requirements must be met. In VR no commonly accepted interaction standards have been defined yet (Hoppe, 2016; Malaika, 2015). Analogous to smartphone gestures development the ongoing VR development will show which emerging interaction paradigms will succeed (Malaika, 2015). Table 1 summarizes some common interaction paradigms.

In general, commonly accepted usability principles like Fitt's Law provide helpful design guidelines. Fitt's Law states user interface requirements for fast and purposeful movements. A study by (Hoppe, 2016) shows that the UI must be quick, intuitive and easy to work with. We adopted the study's strategies in our concept. A special focus is on the workplace suitability, i.e., wearisome sweeping gestures risk fatigue, or the UI must be easy to find and use.

In our concept we focus on the HTC Vive hardware with two controllers. For the interaction with the VID the principles from gesture and smartphone interaction are followed. Display translation can be done via pointing a virtual "laser pointer" and using a controller button dedicated for "left-click" and moving the controller in the designated direction. This is similar to smartphone interaction for moving the display content with one finger. The operations for zooming/scaling and rotating are analogous, i.e., moving controllers apart for zooming, and rotating one controller around a fixed controller for rotation.

The tools must always be ready and easy to use. We propose to adopt the interaction-cube idea from Google Tilt Brush ${ }^{4}$. It should provide easy access to the most common operations, like layer selection, image or scenario reset, contextual assistance.

Table 1. Overview of some common interaction concepts from arbitrarily selected example VR applications

\begin{tabular}{lll}
\hline Concept & Manifestation & Example \\
\hline Locomotion & & \\
\hline With teleportation & Point-to-teleport & Vivecraft \\
& Teleportation with sphere & The Lab \\
\hline Without teleportation & No teleportation & Keep Talking \\
& Float using touchpad & Google Earth VR \\
& Walk using touchpad or controller & The Lab \\
& Walk (room-scale) & \\
\hline Interaction With Images & & Keep Talking \\
\hline Zoom & Head movements & World of Comenius \\
& Stepwise by area selection & Batman VR \\
\hline Pan / move & Dragging finger on touchpad & Google Earth VR \\
& Press and drag & VR Cross View \\
\hline Manipulations & Head movements & \\
\hline Extended functionality & One hand activates functions for other hand & Tilt Brush \\
& Separate console & Subnautica \\
\hline Text input & Speech recognition & Starship Cmd. VR \\
& Large virtual keyboard & Onward \\
\hline Object manipulation & Controller grip and movement & Nvidia Funhouse \\
\hline
\end{tabular}

\section{APPLICATION}

We have verified our concept in a prototype which we implemented using the Unity Game Engine (Figure 4). For the VR integration with the HTC Vive hardware we used Steam VR and the feature rich Virtual Reality Toolkit $^{5}$ (VRTK). To increase the immersion level we implemented the elements of the VIEW concept. This includes scenery, display and interaction paradigms. Gamification aspects involve the integration of assets

\footnotetext{
${ }^{4}$ www.tiltbrush.com, 3D painting VR application useable with motion controllers.

${ }^{5} \mathrm{https} / / / \mathrm{vrtoolkit}$.readme.io, a collection of scripts for Unity3D for VR development.
} 
from a serious game for image interpretation (Atorf et al., 2019). We adopted the original game's science fiction setting which plays in space in a far future. The virtual image interpretation display is placed on the game's spaceship bridge, but whilst the original game itself has a fixed camera position, in VIEW the player is able to move around and play with various elements of the virtual bridge. Lighting and arrangement of the assets are congruent to the original game that has been developed in close cooperation with the target group of image interpreters. To further increase immersion, we added ambient "sci-fi bridge" background sounds. The virtual image display is curved to completely match the field of vision of the players - if they stay directly in front of it. To fulfill fidelity requirements the virtual display lighting is uniform in neutral white, i.e., no spots, no colored light, no shadows. Especially for radar images this is of high importance because of the black-white nature of the image material.

As explained in the VIEW concept we decided against a 360 degree virtual display because of the increased movements efforts and risk of fatigue. This also holds true for a virtual dome. The virtual display is a curved texture with a slippy map image tiling approach. When the user interacts with the texture, it is modified accordingly, e.g., translation, rotation or scaling. The use can select the type of image on the interaction cube, i.e., switch between the various layers of the underlying mapserver to display different image channels for the displayed map location. In the VIEW prototype users can compare three different image channels, i.e., select between a vector map from OpenStreetMap.org, a high-resolution optical satellite image, and a radar image (Figure 5). In our example setting each image channel displays the same location, the Aswan High Dam in South Egypt (Figure 5). One of the challenges for realizing the prototype was to find usable image material from the same location that met the quality requirements (e.g., up-to date or almost identical recording time, high resolution, integrity, etc.). For gamification, the image interpretation tasks are aligned to a storyline which deals with the Aswan High Dam and sabotage actions of fictional rebels. Users must solve image interpretation tasks, e.g., locate rebel camps or analyze road networks, to help the local authorities capture the rebels. In the training scenario additional challenges have been introduced to adjust the difficulty and cover all learning objectives, e.g., clouded high-resolution optical images which prompt the users to switch to radar imagery which is not affected by atmospheric conditions.
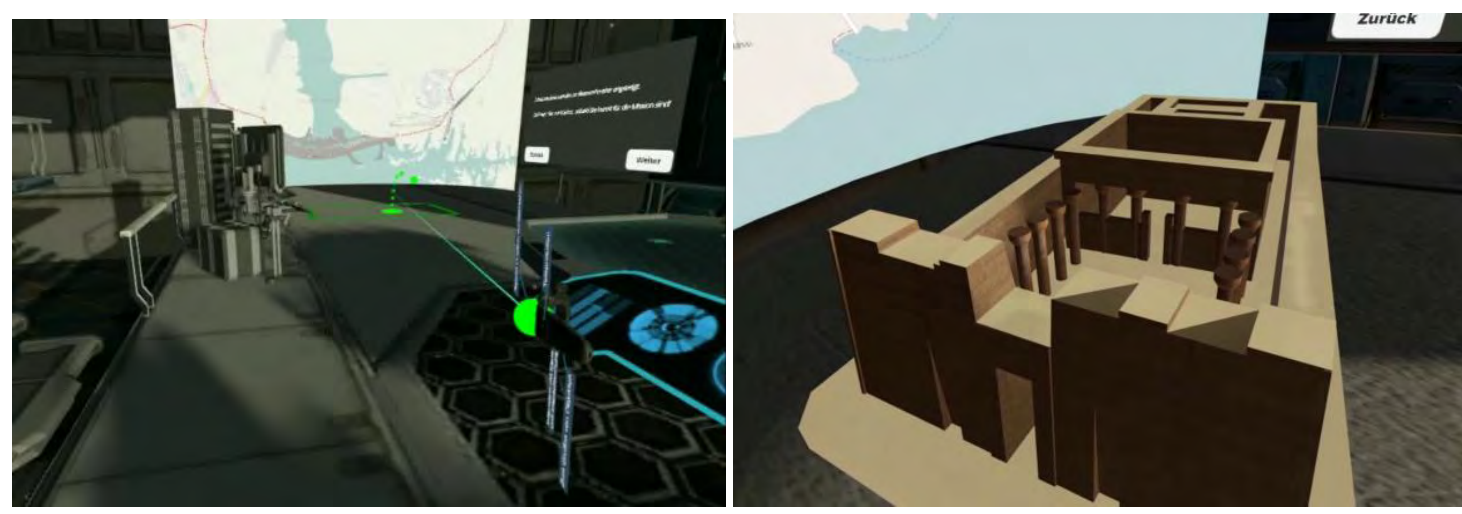

Figure 4. (left) VIEW prototype with bridge scene, teleportation interaction, and curved display for maps and images. (right) 3D model interaction mode
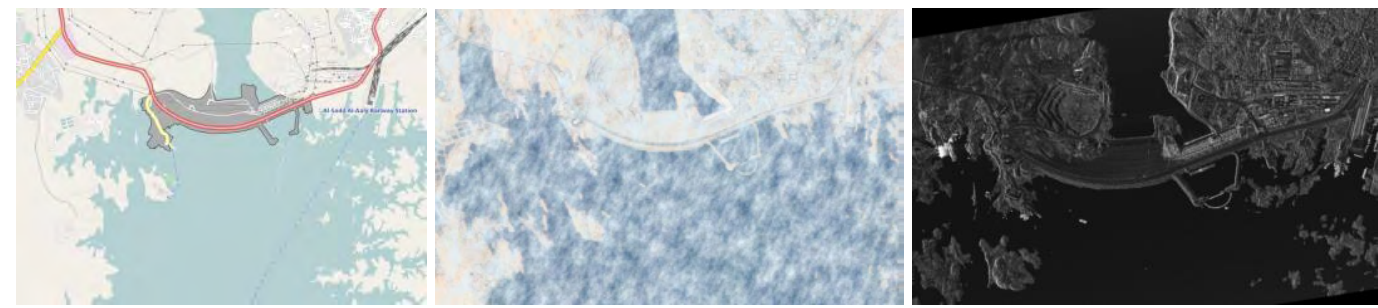

Figure 5. Same location, Aswan High Dam, different image channels: (left) vector map, (center) clouded optical image, (right) radar image 


\section{DISCUSSION}

A preliminary evaluation of the VIEW prototype with $n=16$ users (average age 35,14 male) showed an overall positive acceptance rate and good presence level. The game elements have been evaluated positively and as beneficial for immersion. Also positively evaluated were the 3D modes (Figure 4, right), the different image layers and that the user always is in control. Of negative impact was the grip interaction; the location of the button on the HTC Vive controller was unclear to the participants. Table 2 shows positive and negative statements (translated from German). The results indicate that image interpretations can benefit from VR by increased immersion (e.g., by gamification or spatial presence) and by experiencing spatial content or 3D objects in a more natural way.

Table 2. Positive and negative responses to VIEW

\begin{tabular}{|c|c|}
\hline Positive & Negative \\
\hline $\begin{array}{l}\text { Ifelt comfortable on the bridge. I'd very much } \\
\text { wanted to continue walking there all the time. }\end{array}$ & $\begin{array}{l}\text { My hands are not to be seen, that would be cool-I do } \\
\text { not know if that's possible. }\end{array}$ \\
\hline $\begin{array}{l}\text { The game elements were fun and motivated to } \\
\text { carry on with the task. }\end{array}$ & $\begin{array}{l}\text { The sound does create a higher immersion, but I'd like } \\
\text { to have my rest at work. }\end{array}$ \\
\hline $\begin{array}{l}\text { Interaction with map was super! Scaling as intuitive } \\
\text { as expected. It was super that the map got caught } \\
\text { exactly on the [pointing] ray. }\end{array}$ & $\begin{array}{l}\text { Grip is difficult because the button on the controller is } \\
\text { placed oddly. }\end{array}$ \\
\hline
\end{tabular}

A systematic evaluation is going to study the VIEW concept along the dimensions user acceptance, immersion, image quality recognition, and image interpretation effectivity and efficiency (including usability). The hypothesis is that VIEW offers effective and immersive support for image interpretation tasks and helps in training image interpreters. The questionnaires of interest for us are the Igroup Presence Questionnaire (IPQ), the System Usability Scale (SUS) score (Brooke, 1996), and RawTLX to measure workload. A Web-browser 2D replica is going to serve as a reference model. The replica will mimic most of the features of the VR prototype but obviously address presence and immersion at a different level.

\section{CONCLUSION}

This paper presents the concept study for a VR assistance system for image interpretation, the Virtual Reality Image Exploitation Workspace (VIEW). The application field is aerial image interpretation, i.e., detection, recognition, identification and analysis of structures and objects, according to a given task based on optical, infrared or radar imagery. The concept study explored various approaches for virtual displays as well as interaction paradigms matching the demands for professional image interpretations. Our concept proposes a virtual environment enriched with game elements to increase the immersion level. To display high-resolution imagery data a software architecture for tiled rendering is proposed. Gamification elements like real assets from a congruent serious game, an instruction story, or the possibility to explore the environment promote playability and immersion. The architecture includes a slippy map module and a server for geospatial data, e.g., the open source mapserver software. This approach allows including interoperable image data, labels, annotations, etc. from various sources.

\section{ACKNOWLEDGEMENT}

The underlying project to this article is funded by the Federal Office of Bundeswehr Equipment, Information Technology and In-Service Support under promotional references. The authors are responsible for the content of this article. 


\section{REFERENCES}

Anthes, C., Garcia-Hernández, R.J., Wiedemann, M., Kranzlmüller, D., 2016. State of the art of virtual reality technology. Aerospace Conference. pp. 1-19.

Atorf, D., Kannegieser, E., Roller, W., 2019. Balancing Realism and Engagement for a Serious Game in the Domain of Remote Sensing. In: Söbke H. Gentile M., A.M. (Ed.), Games and Learning Alliance. Springer International Publishing, Cham, pp. 146-156.

Billen, M.I., Kreylos, O., Hamann, B., Jadamec, M.A., Kellogg, L.H., Staadt, O., Sumner, D.Y., 2008. A geoscience perspective on immersive 3D gridded data visualization. Computers and Geoscience. 1056-1072.

Brooke, J., 1996. SUS - A quick and dirty usability scale. Usability Evaluation in Industry. 189, 4-7.

Connolly, T.M., Boyle, E.A., MacArthur, E., Hainey, T., Boyle, J.M., 2012. A systematic literature review of empirical evidence on computer games and serious games. Computers \& Education.

Cronin, B., 2015. The hierarchy of needs in virtual reality development. Medium.com, URL https://medium.com/ @ beaucronin/the-hierarchy-of-needs-in-virtual-reality-development-4333a4833acc.

Dörner, R., Göbel, S., Effelsberg, W., Wiemeyer, J., 2016. Serious Games - Foundations, Concepts and Practice. Springer International Publishing, Cham.

Freina, L., Ott, M., 2015. A literature review on immersive virtual reality in education: State of the art and perspectives. Proceedings of eLearning and Software for Education (eLSE)(Bucharest, Romania, April 23--24, 2015).

Furumura, T., Chen, L., 2004. Large scale parallel simulation and visualization of 3D seismic wavefield using the earth simulator. CMES - Computer Modeling In Engineering And Sciences 6, 153-168.

Ganbatte DevBlog, 2018. Analysing the VR market based on 2017 Steam data. ganbattegame.com. URL https://ganbattegame.com/blog/2018/01/14/analysing-the-vr-market-based-on-2017-steam-data/.

Hoppe, A.H., 2016. Interaktionskonzepte für WIMP-Applikationen in Virtual Reality. KIT.

Hubona, G.S., Shirah, G.W., 2005. Spatial Cues in 3D Visualization. In: Ambient Intelligence for Scientific Discovery. Springer Berlin Heidelberg, pp. 104-128.

Lackey, S.J., Salcedo, J.N., Szalma, J.L., Hancock, P.A., 2016. The stress and workload of virtual reality training: the effects of presence, immersion and flow. Ergonomics 0139, 1-13.

Lin, C.-R., Loftin, R.B., 1998. Application of Virtual Reality in the Interpretation of Geoscience Data. In: VRST '98 Proceedings of the ACM Symposium on Virtual Reality Software and Technology. ACM Press, New York, New York, USA, pp. 187-194.

Loftin, R.B., Nelson, H.R., 2000. Interaction with geoscience data in an immersive environment. In: Proceedings IEEE Virtual Reality 2000 (Cat. No.00CB37048). IEEE Comput. Soc, pp. 55-62.

Malaika, Y., 2015. GDC Vault - Interaction Design in VR: The Rules Have Changed (Again). GDC Europe 2015.

Mazuryk, T., Gervautz, M., 1996. Virtual reality-history, applications, technology and future. Virtual Reality.

Nomura, J., Sawada, K., 2001. Virtual reality technology and its industrial applications. Annual Reviews in Control 25, pp 99-109.

Ohno, N., Kageyama, A., 2007. Scientific visualization of geophysical simulation data by the CAVE VR system with volume rendering. Physics of the Earth and Planetary Interiors 163, 305-311.

Prensky, M., 2001. True Believers: Digital Game-Based Learning in The Military. Digital Game-based Learning. 1-18.

Riva, G., Davide, F., IJsselsteijn, W.A., 2003. Being there: Concepts, effects and measurements of user presence in synthetic environments. Ios Press.

Roller, W., Berger, A., Szentes, D., 2013. Technology based training for radar image interpreters. 6th International Conference on Recent Advances in Space Technologies (RAST). IEEE, pp. 1173-1177.

Streitz, N.A., Tandler, P., Müller-Tomfelde, C., Konomi, S., 2001. Roomware - Towards the Next Generation of Human-Computer Interaction Based on an Integrated Design of Real and Virtual Worlds. Human-Computer Interaction in the New Millennium. Pp. 553-578. 